\title{
Impacto de la realidad aumentada en el rendimiento académico de los estudiantes de educación primaria en la enseñanza de las ciencias naturales
}

\section{The impact of augmented reality on elementary school students' academic performance in teaching natural sciences}

DOMÍNGUEZ-GUTU, Jesús $\dagger^{*}$, GORDILLO-ESPINOZA, Emmanuel, TREJO-TREJO, Gilberto Abelino y CONSTANTINO-GONZÁLEZ, Fernando Exiquio

Universidad Tecnológica de la Selva, México.

ID 1 ${ }^{\mathrm{er}}$ Autor: Jesús, Domínguez-Gutu / ORC ID: 0000-0001-8025-6089, CVU CONACYT ID: 524210

ID $1^{\text {er }}$ Coautor: Emmanuel, Gordillo-Espinoza / ORC ID: 0000-0002-2467-8209, CVU CONACYT ID: 657274

ID $2^{\text {do }}$ Coautor: Gilberto Abelino, Trejo-Trejo / ORC ID: 0000-0003-2808-3939, CVU CONACYT ID: 334014

ID $3^{\text {er }}$ Coautor: Fernando Exiquio, Constantino-González / ORC ID: 0000-0002-9701-1990, CVU CONACYT ID: 79617

DOI: $10.35429 /$ JOTE.2020.12.4.1.12

Recibido 10 de Abril, 2020; Aceptado 30 de Junio, 2020

Resumen

La realidad aumentada es una tecnología que permite combinar la vida real con información digital, a través de otras tecnologías como las computadoras, tablets o smartphones. Actualmente, se utiliza en diversas áreas como la publicidad, medicina, artes, educación, entre otras. Esta investigación se realizó en la ciudad de Ocosingo, Chiapas, México, en un contexto donde es difícil tener acceso a la tecnología y sobre todo a la conectividad, se centró en utilizar a la realidad aumentada en una secuencia didáctica para el proceso de enseñanza y aprendizaje de los aparatos circulatorio, respiratorio y digestivo, de la materia de Ciencias Naturales, con el fin de demostrar si el uso de esta tecnología como una herramienta didáctica, permite a los estudiantes del $4^{\circ}$. grado de primaria mejorar su rendimiento académico. El estudio se efectuó bajo un enfoque cuantitativo cuasiexperimental y descriptivo, utilizando como herramientas de recolección de datos, pruebas diseñadas con respuestas dicotómicas (pre-test y pos-test) y encuestas con preguntas cerradas bajo la escala de Likert. Los resultados demuestran que usar la realidad aumentada dentro del aula de clases en el proceso de enseñanza y aprendizaje, incrementa significativamente el rendimiento académico de los estudiantes, comparado con la enseñanza tradicional de éstos temas en la materia mencionada.

Realidad aumentada, Secuencia didáctica, Rendimiento académico, Educación primaria, Ciencias naturales

\section{Abstract}

Augmented reality is a technology that allows combining realworld life with digital information, through other technologies such as computers, tablets or smartphones. Currently, it is used in diverse areas such as advertising, medicine, arts and education, among others. This research was carried out in the city of Ocosingo, Chiapas, Mexico, in a context where it is difficult to access technology and, above all, connectivity. It focused on using augmented reality in a didactic sequence for the teaching and learning process of the circulatory, respiratory and digestive systems in the Natural Sciences subject, in order to demonstrate whether the use of this technology as a didactic tool allows 4th grade students to improve their academic performance. The study was carried out under a quantitative, quasi-experimental and descriptive approach, using data collection tools such as tests designed with dichotomous responses (pre-test and post-test) and surveys with closed-ended questions under the Likert scale. The results show that using augmented reality in the teaching and learning process, within the classroom, significantly increases the academic performance of students compared to the traditional way of teaching these topics in the aforementioned subject.

Augmented reality, Didactic sequence, Academic performance, Elementary school, Natural sciences

*Correspondencia al Autor (Correo Electrónico: jdominguez@laselva.edu.mx)

$\dagger$ Investigador contribuyendo como primer autor. 


\section{Introducción}

Al paso de los años, las Tecnologías de la Información y Comunicación (TIC) han tenido presencia en diversas áreas como la Economía, Finanzas, Negocios, Mercadotecnia, Industrias, Educación, entre otras. En el sector educativo, se ha aplicado en áreas como la medicina, biología, química, geometría, física, matemáticas, astronomía, historia, etc. (Hamiyet y Rabia, 2019).

Algunos países de Europa, Asia y América han modificado sus políticas educativas para su incorporación en los procesos educativos, con el fin de implementar estrategias didácticas y pedagógicas innovadoras que apoyen el aprendizaje y el desarrollo de competencias.

En México, la implementación de las TIC en la educación ha sido un proceso muy lento, sin embargo, existen Universidades que han implementado diversas tecnologías en el aula como apoyo al proceso de enseñanza y aprendizaje, tales como, herramientas Web 3.0, plataformas LMS (Learning Management System), entre otras; caso contrario en la educación básica (primaria y secundaria) y media superior, donde la resistencia de los docentes al uso de las TIC y la falta de infraestructura tecnológica en éstos niveles educativos (Domínguez y Sandoval, 2017), han dificultado esta implementación.

La incorporación de las tecnologías de la información y comunicación como apoyo al proceso de enseñanza y aprendizaje están empezando a ser utilizadas dentro de las aulas. Los docentes tienen que buscar nuevas estrategias que permitan despertar el interés en los alumnos por las materias que imparten.

Dentro de estas herramientas tecnológicas se encuentra la realidad aumentada conocida como RA, siendo una tecnología que ha sido ampliamente utilizada por los países de primer mundo y se utiliza en las áreas de medicina, música, historia, educación, entre otras.
En la actualidad, algunas instituciones de educación superior y medio superior están incursionado en la utilización de nuevas tecnologías en el aula como la realidad aumentada, a través del uso de dispositivos como tablets y smartphones, para la manipulación de aplicaciones de software que ayudan a los estudiantes a adquirir nuevas habilidades y conocimientos, permitiéndoles aprender en entornos difíciles de contextualizar dentro del aula de clases de manera tradicional.

Mirete (2010) menciona que el uso de la tecnología en las aulas se ha convertido en una realidad, pero presentan obstáculos como la falta de cultura y alfabetización digital, además de una inadecuada formación docente para la implementación de las TIC (p. 36). Avendaño (2015) afirma que "de un $82 \%$ de docentes que siguen utilizando el método de enseñanza de pizarrón, en primaria el $51 \%$ de los maestros y en secundaria el $68 \%$ afirma que muy frecuentemente exponer la clase para que después los alumnos tomen nota dando a entender que se vuelve a recaer en el método tradicional de enseñanza" (p. 41); por lo que, en la educación básica (primaria y secundaria), no se ha aprovechado la tecnología existente para innovar el proceso de enseñanza y aprendizaje.

\section{Zorro, Torres y Rivera (2019)}

mencionan "La realidad aumentada es una tecnología que puede ayudar a los procesos pedagógicos y didácticos en las aulas de clase, debido a la gran aceptación que tienen las tecnologías de información y comunicación entre los estudiantes contemporáneos" (p. 15), esta tecnología permite plantear situaciones dentro del aula que tradicionalmente no se podría hacer, como en el caso de la materia de Ciencias Naturales, específicamente en la enseñanza del cuerpo humano, que permite demostrar cómo funcionan los diferentes aparatos (circulatorio, digestivo, respiratorio, etc.) y los órganos que lo conforman, detallando su forma, color y textura de una manera visual, permitiendo al estudiante descubrirlos de manera interactiva. 


\section{Marco Teórico}

La realidad aumentada se empezó a utilizar desde la década de los 60's en el área militar y la medicina, hoy en día, se presenta en diversas áreas como la publicidad, geolocalización, educación, entre otras. (Bursali y Yilmaz, 2019). Diversos autores han definido la Realidad Aumentada, Azuma (1997) menciona que "un sistema de RA debe contener las siguientes características para considerarse como tal: combinar la realidad y lo virtual, ser interactivo en tiempo real y ser registrado en forma de 3D" (p. 356); Van Krevelen y Poelman (2010) mencionan la "RA complementa el mundo real con objetos virtuales creados por una computadora, que parecen coexistir en el mismo espacio que el mundo real" (p. 1).

Por lo que, la Realidad Aumentada permite combinar la vida real con información digital, a través de otras tecnologías como las computadoras, tablets o smartphones que permiten visualizar una nueva realidad donde se integran textos, videos, sonidos, entre otros, que posibilitan la interactividad entre todos estos elementos.

\section{La realidad aumentada en la educación}

En países de Europa, han realizado estudios en el sector educativo usando la realidad aumentada, en la Universidad Nacional de Educación a Distancia en España, utilizaron la realidad aumentada como recurso didáctico en el área de las ciencias sociales, específicamente en la presentación de contenidos de historia del arte (Sáez, Cózar y Domínguez, 2018). En la Universidad de Córdoba en España, Marín (2018) realizó un estudio para determinar si el uso de la realidad aumentada mejora la educación inclusiva en la educación primaria, tanto en los estudiantes como en los docentes; en ésta última, también realizaron un estudio para incluir a la realidad aumentada en el aula infantil como una innovación docente, a través de la aplicación ChromVille para el aprendizaje del cuerpo humano (Marín y Muñoz, 2018). En Turquía, realizaron un estudio para determinar los niveles de comprensión lectora, permanencia del aprendizaje y actitudes de los estudiantes de secundaria, a través de la lectura con materiales de realidad aumentada. (Bursali y Yilmaz, 2019)
En países de Latinoamérica, en la Corporación Universitaria Minuto de Dios de Colombia, realizaron un estudio donde utilizaron la realidad aumentada para evaluar su efecto en el rendimiento académico de los estudiantes de educación básica, diseñaron una experiencia didáctica en el tema de las regiones naturales de ese país en la asignatura de Ciencias Sociales (López y Gutiérrez, 2018). En la Universidad Pedagógica y Tecnológica del mismo país, aplicaron la realidad aumentada a través de la aplicación Arloon Anatomy como apoyo de la enseñanza de las ciencias naturales en la educación primaria, diseñando una secuencia didáctica para el proceso de enseñanza y aprendizaje del aparato digestivo (Angarita, 2018).

En México, en la Universidad Autónoma de Ciudad Juárez, desarrollaron una aplicación que usa la realidad aumentada para el aprendizaje del tiro parabólico de la materia de física, aplicado en estudiantes de educación superior (Parroquín et al., 2013). En la Universidad Tecnológica del Centro de Veracruz, desarrollaron un simulador virtual implementando la realidad aumentada, a través del desarrollo de marcadores que representaban a los elementos químicos de la tabla periódica, instrumentos de laboratorio y diferentes materiales que se usan en los laboratorios de química (Zarate et al., 2013).

La UNAM en colaboración con la Universidad Católica de Valparaíso de Chile, realizaron un estudio sobre el diseño de secuencias de enseñanza y aprendizaje para la materia de química, utilizando la realidad aumentada (Merino et al., 2015). En Instituto Tecnológico de Oaxaca, desarrollaron una aplicación móvil para el aprendizaje del idioma inglés utilizando la realidad aumentada dirigida esencialmente a niños (Morales et al., 2015). En la Universidad Autónoma de Chihuahua, desarrollaron una aplicación con realidad aumentada para la enseñanza de cuerpos geométricos en la educación primaria (Carrillo y Cortés, 2016). En la Universidad Autónoma de Querétaro, desarrollaron una aplicación con realidad aumentada para tabletas y smartphones, con el fin de crear conciencia en la sociedad para la conservación de la biodiversidad de las áreas naturales de Querétaro (Pérez et al., 2016). 
En el Instituto Politécnico Nacional, desarrollaron una aplicación móvil de RA como complemento didáctico en el área de Geometría en la materia de Cálculo del nivel medio superior (Gómez, Medel y García, 2018).

Como se observa, la Realidad Aumentada representa actualmente una potente herramienta que ha mostrado su versatilidad en un amplio abanico de aplicaciones en diferentes áreas de conocimiento, donde ha encontrado grandes posibilidades para la difusión y conocimiento de contenidos que se presenta de una forma atractiva y pedagógica al mismo tiempo (Ruiz, 2011), sin embargo, son muy pocas investigaciones que han estudiado a la RA en el área de las ciencias naturales en la educación primaria.

\section{Aplicaciones de realidad aumentada}

En la actualidad, existen diversas aplicaciones de RA para la enseñanza de las ciencias naturales, específicamente del cuerpo humano, entre ellas, Chromville y Arloon Anatomy. Chromville son una serie de aplicaciones que se puede utilizar en educación primaria, básicamente para primer y segundo grado, en el cual los estudiantes pueden colorear los diversos dibujos existentes que fungen como marcadores para tablets o smartphones, para trabajar con los estudiantes el tema que se requiera.

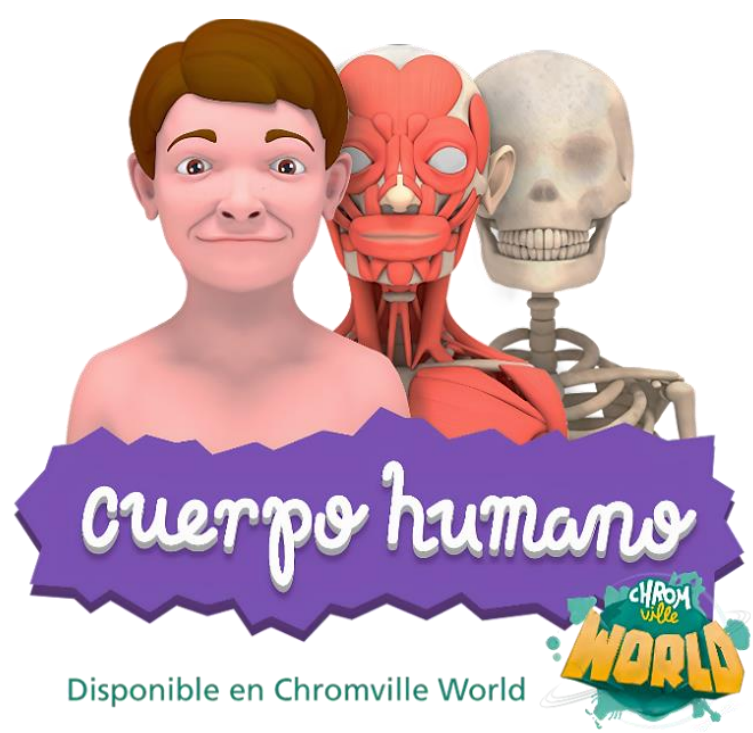

Figura 1 Chromville cuerpo humano Fuente: Chromville (2015), obtenido de
https://chromville.com/chromvillesciencel

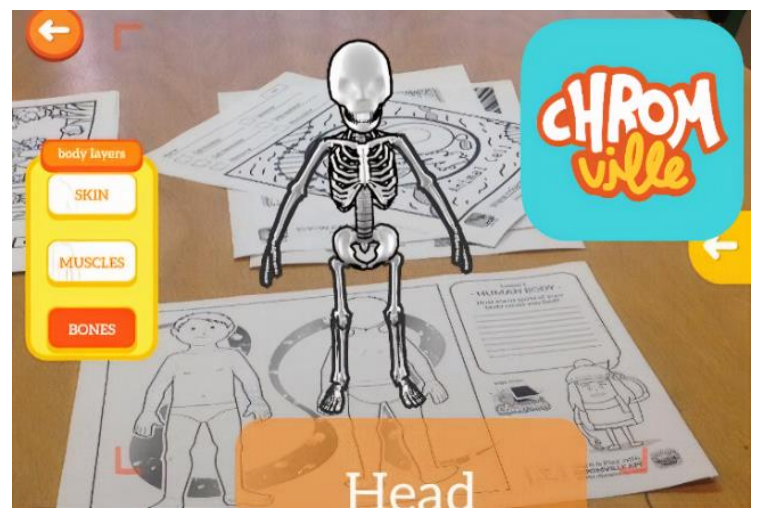

Figura 2 Marcador de Chromville cuerpo humano Fuente: Chromville (2015), obtenido de https://chromville.com/2016/03/01/5-reasons-why-arshould-be-used-by-every-child/

Arloon Anatomy, es una aplicación más desarrollada que la anterior, que puede utilizarse desde tercer grado de primaria, inclusive hasta la secundaria, debido al detalle que presenta en cada uno de los aparatos del cuerpo humano, esta aplicación, maneja contenido audiovisual y se trabaja mediante marcadores donde los estudiantes interactúan con la aplicación.

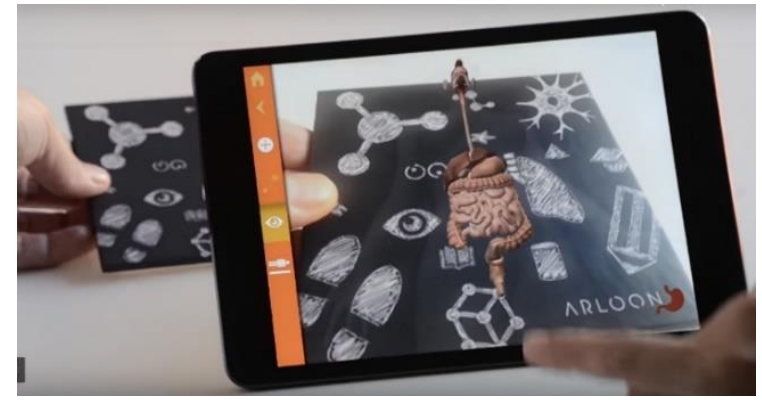

Figura 3 Arloon Anatomy

Fuente: Arloon (2019), obtenido de http://www.arloon.com/apps/arloon-anatomy/

\section{Rendimiento académico}

Jiménez (2000) define al rendimiento académico como un nivel de conocimientos demostrado en un área o materia comparado con la norma de edad y nivel académico, sin embargo, de acuerdo con Osorio (2012) la idea que se tiene actualmente sobre el rendimiento académico, es una calificación obtenida en un examen de conocimientos al que es sometido un alumno, únicamente relacionado al aspecto intelectual. 
Osorio (2012), menciona que el rendimiento escolar se ve afectado por una serie de factores, éstos pueden ser divididos en dos aspectos: en el entorno familiar, la desatención de los padres que demuestran poco interés en sus hijos al dejarle la responsabilidad de su formación a la escuela y al docente, impactando en la conducta de éstos que se ve reflejado en su bajo rendimiento académico; los hogares destruidos (divorcios), cambian la conducta del estudiantes, al carecer de atención en su hogar por la usencia de uno de los padres; el nivel socioeconómico, afecta el rendimiento del estudiante por la falta de apoyo económico para la realización de actividades en casa, escasos recursos didácticos para el aprendizaje como libros, diccionarios, enciclopedias, computadora e internet, ha impactado en la entrega de tareas extraescolares, aunque no aplica para todos, ya que existen estudiantes de bajos recursos económicos muy inteligentes y estudiantes con todos los recursos didácticos disponibles que no tienen el mejor desempeño en la escuela.

Por otro lado, en el entorno escolar, el impacto del bajo rendimiento académico de los estudiantes es originado por el compromiso del docente en la formación de éstos, la falta de innovación en el proceso de enseñanza o el desinterés por preparar su tema, puede ocasionar el desinterés del estudiante por aprender; otro factor es la infraestructura escolar, la falta de bibliotecas, salas de cómputo, energía eléctrica, sanitarios, malas condiciones de las aulas, etc., ha impactado notablemente en el rendimiento escolar, sobre todo en las zonas rurales e indígenas donde la infraestructura escolar es deficiente.

Aunque existen políticas públicas que atacan los diferentes aspectos mencionados, aún existe inequidad en el otorgamiento de los apoyos por parte de las autoridades gubernamentales en las zonas rurales, para el mejoramiento de la calidad de la educación en el país.

\section{Planteamiento del problema}

En México, se ha implementado el uso de las TIC en el sector educativo, a través de la oferta educativa a nivel superior, con el uso de plataformas educativas, así como la oferta de cursos online masivos y abiertos $\left(\mathrm{MOOC}^{1}\right)$ como formación profesional y empresarial. En educación básica, se implementó Enciclomedia como una iniciativa del Gobierno Federal para implementar las TIC en el salón de clases, con la consulta de libros de texto gratuitos que tenían relación con los contenidos del programa oficial de estudios, la versión 1 en el 2003, la versión 1.2 en el 2004 y la versión 2 en el sexenio del Lic. Felipe Calderón Hinojosa. (Hernández y Bautista, 2017)

En el 2013, el Gobierno Federal implementó el Programa de Inclusión y Alfabetización Digital denominado micompu.mx, que buscaba impactar en el proceso de enseñanza - aprendizaje al incluir computadoras e internet en las escuelas de nivel primaria para 5o. y 6o. grado. (Linares, 2014)

Sin embargo, como concluye Edgar Vásquez (s.f.) en su artículo "Las TIC en la educación pública de México, un esfuerzo disparejo", en el portal u-gob.com:

"Aunque el esfuerzo que el gobierno mexicano ha hecho para utilizar las TIC para mejorar la educación pública es loable, me queda la impresión de que lo hecho es aún pequeño para el tamaño de país que somos y que en la elaboración de contenidos educativos no sólo prevalece un criterio centralista, sino que al mismo tiempo no utiliza las posibilidades del crowdsourcing o inteligencia colectiva, pues si se involucrará a alumnos y maestros de todo México la producción de material pedagógico crecería exponencialmente al tiempo que se ahorraría tiempo y recursos tanto humanos como económicos."

\footnotetext{
${ }^{1}$ MOOC acrónimo en inglés de Massive Online Open Courses
}

DOMÍNGUEZ-GUTU, Jesús, GORDILLO-ESPINOZA, Emmanuel, TREJO-TREJO, Gilberto Abelino y CONSTANTINO-GONZÁLEZ, Fernando Exiquio. Impacto de la realidad aumentada en el rendimiento académico de los estudiantes de educación primaria en la enseñanza de las ciencias naturales. Revista de Educación Técnica. 2020 
Chiapas ocupa el primer lugar a nivel nacional en rezago educativo con el $47.9 \%$ de su población con edad de 15 años en adelante, que no sabe leer y escribir o que no concluyó la primaria o secundaria. En el Plan de Desarrollo Estatal 2019 - 2024 menciona que, uno de los principales problemas de la educación básica es el abandono escolar, esto obedece a diversas causas como la falta de interés por estudiar o asistir a clases, motivando al deficiente rendimiento escolar que muestran los alumnos; por otro lado, y no menos importante, el poco interés en la profesionalización del docente y la falta de propuestas innovadoras en el proceso de enseñanza - aprendizaje, incrementan este problema. (Secretaría General de Gobierno, 2019).

\section{Objetivo}

Atendiendo a una de las problemáticas existentes en el estado de Chiapas, los investigadores se centraron en implementar la realidad aumentada como una innovación en el proceso de enseñanza y aprendizaje, teniendo como objetivo de esta investigación demostrar que el uso de la realidad aumentada como una herramienta didáctica, permite a los estudiantes del $4^{\circ}$. grado de primaria federal de la cabecera municipal de Ocosingo, mejorar su rendimiento académico en la materia de ciencias naturales, específicamente en el aprendizaje del sistema circulatorio, respiratorio y digestivo.

\section{Metodología}

De acuerdo con Hernández, Fernández y Baptista (2014), esta investigación se realizó bajo un enfoque cuantitativo, en la dimensión 1, se utiliza un diseño cuasiexperimental con un grupo experimental y un grupo de control, teniendo como variable independiente a la realidad aumentada y la variable dependiente al rendimiento académico, para el cual se planteó la hipótesis "La herramienta de realidad aumentada mejora el rendimiento académico de los estudiantes del $4^{\circ}$. grado de primaria federal de la cabecera municipal de Ocosingo, Chiapas"; en la dimensión 2, se utiliza un diseño descriptivo para medir la percepción de los estudiantes sobre el uso de esta herramienta.

\section{Muestra}

La muestra de este estudio fue no probabilística e intencional, ésta se determinó con base al rendimiento académico obtenido en el ciclo escolar $2018-2019^{2}$, en la materia de Ciencias Naturales del $3^{\text {er }}$ grado de las escuelas primarias federales de la cabecera municipal de Ocosingo, Chiapas, eligiéndose a 3 escuelas primarias, una con el mejor rendimiento académico, otra con un rendimiento promedio y la otra con el rendimiento más bajo, obteniéndose así un total de 149 alumnos con edades de 9 y 10 años del $4^{\circ}$. grado de primaria, 74 de ellos en el grupo experimental y 75 en el grupo de control, segmentados por género en 78 hombres y 71 mujeres, de ellos, 40 hombres y 34 mujeres son del grupo de experimento, y 38 hombres y 37 mujeres del grupo de control.

\begin{tabular}{|l|r|r|r|}
\hline \multicolumn{1}{c}{ Escuela } & \multicolumn{2}{c}{ Sexo } & Total \\
\cline { 2 - 3 } & Masculino & \multicolumn{1}{c}{ Femenino } & \\
\hline Cuauhtémoc & 31 & 36 & 67 \\
\hline Marcos Villanueva & 19 & 16 & 35 \\
\hline Niños Héroes & 28 & 19 & 47 \\
\hline Total & $\mathbf{7 8}$ & $\mathbf{7 1}$ & $\mathbf{1 4 9}$ \\
\hline
\end{tabular}

Tabla 1 Distribución del género de los alumnos por escuelas

Fuente: Elaboración propia, 2020

\section{Instrumentos de recopilación de información}

En la dimensión 1, se utilizó un pre-test y postest diseñado para el estudio, con respuestas dicotómicas para los aparatos circulatorio, respiratorio y digestivo, con el fin de medir el rendimiento académico de los estudiantes; para medir el impacto de la realidad aumentada, los resultados obtenidos por los estudiantes en las pruebas, se convirtieron en puntajes con escala de 0 a 10, con el fin de realizar una prueba $\mathrm{T}$ de Student.

\footnotetext{
${ }^{2}$ Datos proporcionados por la Delegación Regional en Ocosingo, de la Secretaría de Educación del Estado de Chiapas
}

DOMÍNGUEZ-GUTU, Jesús, GORDILLO-ESPINOZA, Emmanuel, TREJO-TREJO, Gilberto Abelino y CONSTANTINO-GONZÁLEZ, Fernando Exiquio. Impacto de la realidad aumentada en el rendimiento académico de los estudiantes de educación primaria en la enseñanza de las ciencias naturales. Revista de Educación Técnica. 2020 
Para esta dimensión, se calculó la fiabilidad del instrumento dicotómico de manera general, a través del coeficiente de Kuder Richardson (KR20) usando el software estadístico SPSS, arrojando un valor de 0.862 , siendo un instrumento con coeficiente de confiabilidad Muy Alto (Ruíz, 2013); se realizaron las pruebas de confiabilidad KR20 de los instrumentos por separados, los del pre-test arrojan un valor de 0.771 y los del pos-test 0.841 , teniendo un coeficiente de confiabilidad Alto y Muy Alto, respectivamente.

En la dimensión 2, se aplicó una encuesta dividida en dos secciones, la primer sección denominada uso de la tecnología, consta de 4 preguntas dicotómicas, la segunda sección denominada uso de la aplicación, consta de 4 preguntas bajo la escala de Likert, con el fin de medir la percepción de los estudiantes del grupo experimental que utilizaron la herramienta de realidad aumentada.

\section{Desarrollo}

La intervención efectuada en el grupo experimental fue diseñada con una secuencia didáctica centrada en el estudiante, a través de una constante participación durante su proceso de aprendizaje (Ontoria, 2004), ésta estuvo desarrollada en tres fases:

\section{Fase de diseño}

Los investigadores diseñaron la intervención apegado a los contenidos del libro de texto gratuito de la SEP para el $4^{\circ}$. grado de primaria, se realizó la búsqueda de una aplicación de realidad aumentada, encontrando la mejor opción en la aplicación de Arloon Anatomy, que maneja diversos aparatos del cuerpo humano, tales como, respiratorio, circulatorio, digestivo, excretor, nervioso, esquelético, muscular y reproductivo, eligiéndose los tres primeros, que están incluidos en el libro de texto antes mencionado. Se diseñó una presentación para la explicación de cada uno de los aparatos, utilizando imágenes GIF, esquemas, enriqueciéndolos con videos que se encuentran en la aplicación de Arloon Anatomy.

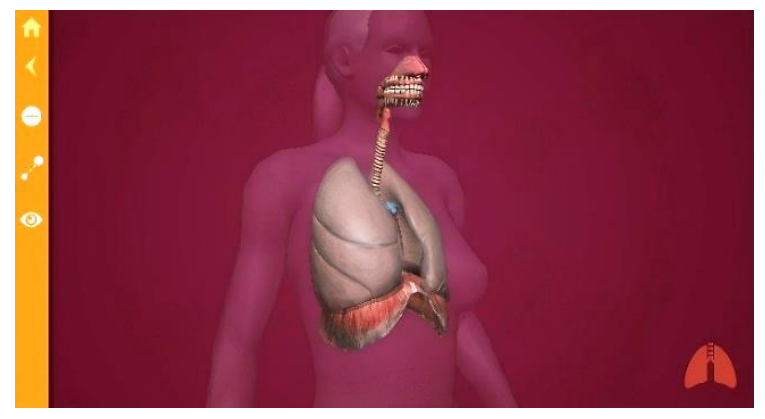

Figura 4 Aplicación de realidad aumentada Arloon Anatomy: Aparato respiratorio

Fuente: Arloon

\section{Fase de intervención}

Antes de realizar la intervención, se aplicó el pre-test a ambos grupos, con el fin de medir los conocimientos previos que tenían en cada uno de los aparatos circulatorio, respiratorio $y$ digestivo; posteriormente, en el grupo de control, el docente procedió a la explicación del tema de manera tradicional, en el grupo experimental los investigadores realizaron la intervención diseñada para el estudio, donde los estudiantes interactuaron con la aplicación de realidad aumentada, a través del marcador de la aplicación. Una vez terminada la intervención, se procedió a aplicar el pos-test para medir los conocimientos adquiridos en ambos grupos.

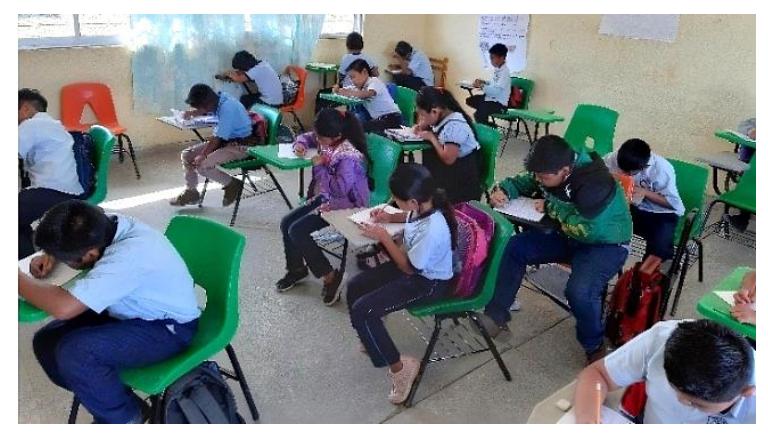

Figura 5 Aplicación del pre-test

Fuente: Elaboración propia, 2020

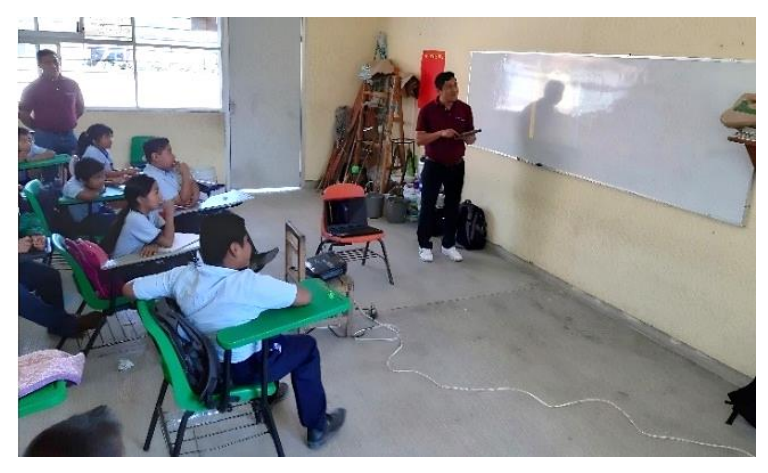

Figura 6 Intervención: Explicación del aparato circulatorio

Fuente: Elaboración propia, 2020 


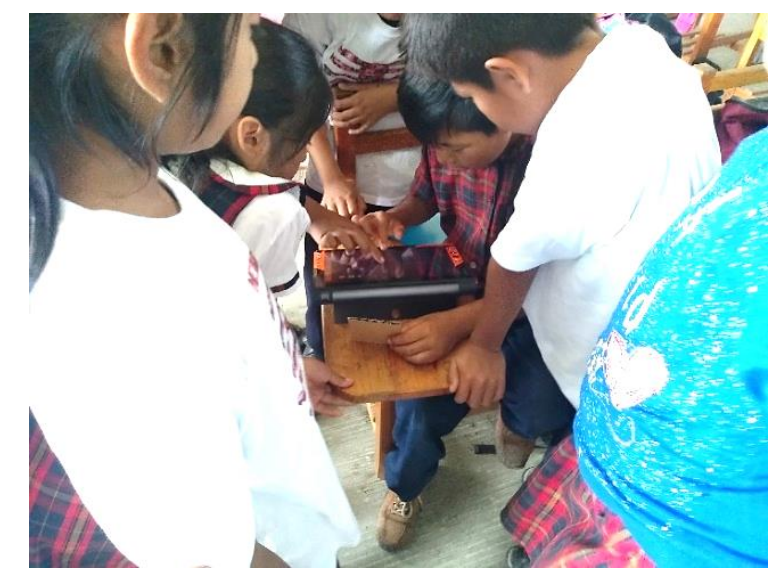

Figura 7 Interacción con la aplicación de realidad aumentada

Fuente: Elaboración propia, 2020

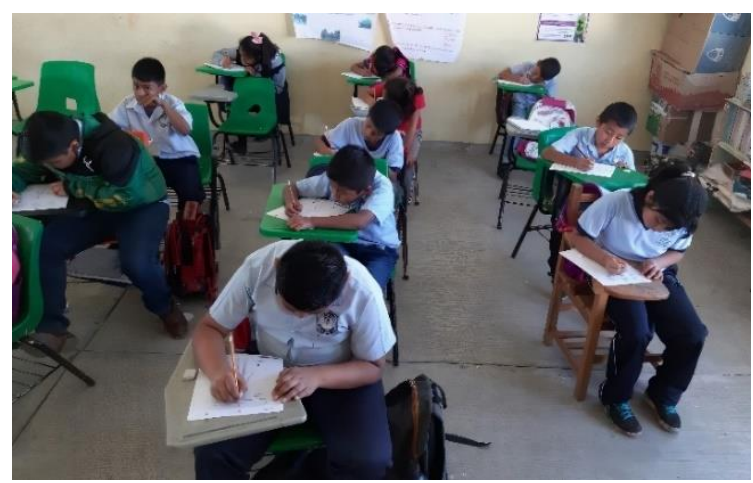

Figura 8 Aplicación del pos-test

Fuente: Elaboración propia, 2020

\section{Fase de Recolección de datos}

Después de la intervención en el grupo experimental, se aplicó una encuesta final para conocer el uso de la tecnología y el uso de la aplicación, con el fin de medir la percepción de los estudiantes que utilizaron la herramienta de realidad aumentada.

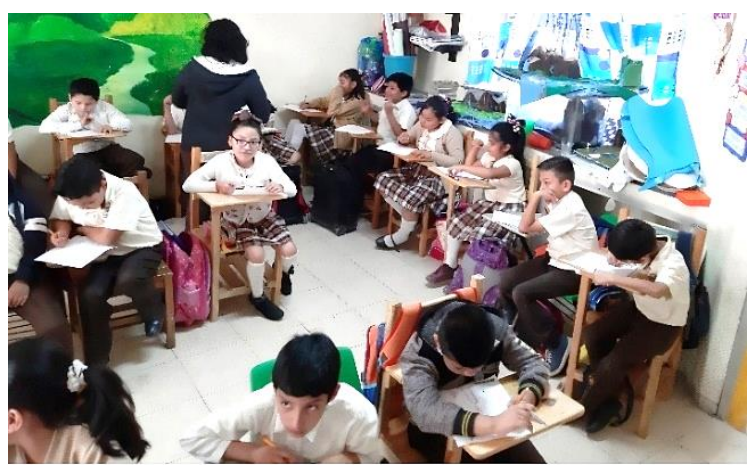

Figura 9 Aplicación de la encuesta final Fuente: Elaboración propia, 2020

\section{Resultados}

\section{Dimensión 1}

Para la determinación de la prueba a utilizar, con el fin de medir el impacto de la herramienta realidad aumentada en el rendimiento académico de los estudiantes, se realizó la prueba de normalidad de Kolgomorov Smirnov, encontrando que los datos son normales, ya que el valor de $p=0.200>\alpha=0.05$. Para probar la hipótesis planteada, se realizó la prueba $\mathrm{T}$ de Student para muestras independientes entre el grupo de experimento y el grupo de control, utilizando el software estadístico SPSS, con un nivel de significancia de $\alpha=0.05$.

Los resultados obtenidos de la prueba $\mathrm{T}$ de Student, arroja que el valor de $\mathrm{p}=0.000$ es menor que el nivel de significancia establecido de $\alpha=0.05$, por lo que, se rechaza la hipótesis nula, ya que los datos sustentan que existe diferencia significativa entre ambos grupos, aceptándose la hipótesis planteada en la investigación.

Se realizó la misma prueba T de Student en los diferentes aparatos (circulatorio, respiratorio y digestivo) del cuerpo humano por separado, obteniéndose en el aparato circulatorio un valor de $\mathrm{p}=0.319$ siendo mayor que el nivel de significancia establecido de $\alpha=0.05$, por lo que, no existe diferencia significativa entre ambos grupos; en el aparato respiratorio, se obtuvo un valor de $\mathrm{p}=0.000$ siendo mayor que el nivel de significancia establecido de $\alpha=0.05$, por lo que, existe diferencia significativa entre ambos grupos; para el aparato digestivo, se obtuvo un valor de $\mathrm{p}=0.000$ siendo mayor que el nivel de significancia establecido de $\alpha=0.05$, por lo que, existe diferencia significativa entre ambos grupos.

Los datos anteriores muestran que, para el aparato circulatorio la herramienta de realidad aumentada no impacta en el rendimiento académico de los estudiantes, mientras que, en los aparatos respiratorio y digestivo, impacta significativamente en su rendimiento. 


\section{Dimensión 2}

En la primera sección uso de la tecnología, de los 74 estudiantes del grupo experimental el 63.5\% cuenta con una Tablet o Smartphone en su casa, el $51.4 \%$ cuenta con Internet en su casa, el $25.7 \%$ mencionó que conocía una aplicación de realidad aumentada como la que usó y el $32.4 \%$ mencionó que había utilizado una aplicación de realidad aumentada para aprender algún tema que vio en la escuela. En la Tabla 2, se puede observar los resultados del uso de la tecnología por escuela, donde se observa que en la escuela seleccionada con el rendimiento académico más bajo (Marcos Villanueva), son muy pocos alumnos que cuentan con un dispositivo móvil e internet en su casa, por lo que para la mayoría fue la primera ocasión que interactuaron con una Tablet y una aplicación de realidad aumentada.

\begin{tabular}{|l|r|r|r|r|}
\hline Escuela & $\begin{array}{c}\text { Tienen un } \\
\text { dispositivo } \\
\text { móvil }\end{array}$ & $\begin{array}{c}\text { Tienen } \\
\text { Internet } \\
\text { en casa }\end{array}$ & $\begin{array}{c}\text { Conocen } \\
\text { una } \\
\text { aplicación } \\
\text { de RA }\end{array}$ & $\begin{array}{c}\text { Han } \\
\text { utilizado } \\
\text { una } \\
\text { aplicación } \\
\text { de RA }\end{array}$ \\
\hline Cuauhtémoc & 26 & 26 & 8 & 10 \\
\hline Marcos Villanueva & 8 & 4 & 6 & 4 \\
\hline Niños Héroes & 13 & 8 & 5 & 10 \\
\hline Totales & $\mathbf{4 7}$ & $\mathbf{3 8}$ & $\mathbf{1 9}$ & $\mathbf{2 4}$ \\
\hline
\end{tabular}

Tabla 2 Uso de la tecnología de los estudiantes por escuela.

Fuente: Elaboración propia, 2020

La percepción del estudiante fue fundamental para conocer si la aplicación de realidad aumentada fue útil, agradable y sencilla de usar, los resultados obtenidos se presentan en la Tabla 3, donde el $82.4 \%$ le gustó usar la aplicación, el $77 \%$ cree que le ayudó a aprender más, el $56.8 \%$ se le facilitó usar la aplicación y 78.4\% le gustaría seguir utilizando este tipo de aplicación para aprender Ciencias Naturales.

\begin{tabular}{|c|c|c|c|c|c|}
\hline Ítem & Muchísimo & Mucho & $\begin{array}{l}\text { Ni si, } \\
\text { ni no }\end{array}$ & Poco & Nada \\
\hline $\begin{array}{lr}\text { ¿Qué tanto te } \\
\text { gustó utilizar esta } \\
\text { aplicación para } \\
\text { aprender r las } \\
\text { partes de } \\
\begin{array}{ll}\text { cuerpo? } & \end{array} \\
\end{array}$ & $\begin{array}{r}61 \\
82.4 \%\end{array}$ & $\begin{array}{r}9 \\
12.2 \%\end{array}$ & $\begin{array}{r}3 \\
4.1 \%\end{array}$ & $\begin{array}{r}0 \\
0.0 \%\end{array}$ & $\begin{array}{r}1 \\
1.4 \%\end{array}$ \\
\hline $\begin{array}{l}\text { Al utilizar esta } \\
\text { aplicación, ¿Qué } \\
\text { tanto te ayudó a } \\
\text { aprender las } \\
\text { partes de tu } \\
\text { cuerpo? }\end{array}$ & $\begin{array}{r}57 \\
77.0 \%\end{array}$ & $\begin{array}{r}12 \\
16.2 \%\end{array}$ & $\begin{array}{r}0 \\
0.0 \%\end{array}$ & $\begin{array}{r}4 \\
5.4 \%\end{array}$ & $\begin{array}{r}1 \\
1.4 \%\end{array}$ \\
\hline $\begin{array}{l}\text { ¿Qué tanto se te } \\
\text { facilitó utilizar } \\
\text { esta aplicación? }\end{array}$ & $\begin{array}{r}42 \\
56.8 \%\end{array}$ & $\begin{array}{r}17 \\
23.0 \%\end{array}$ & $\begin{array}{r}2 \\
2.7 \%\end{array}$ & $\begin{array}{r}11 \\
14.9 \%\end{array}$ & $\begin{array}{r}2 \\
2.7 \%\end{array}$ \\
\hline $\begin{array}{l}\text { ¿Qué tanto te } \\
\text { gustaría } \\
\text { continuar } \\
\text { aprendiendo } \\
\text { Ciencias } \\
\text { Naturales } \\
\text { utilizando una } \\
\text { aplicación como } \\
\text { ésta? } \\
\end{array}$ & $\begin{array}{r}58 \\
78.4 \%\end{array}$ & $\begin{array}{r}7 \\
9.5 \%\end{array}$ & $\begin{array}{r}2 \\
2.7 \%\end{array}$ & $\begin{array}{r}6 \\
8.1 \%\end{array}$ & $\begin{array}{r}1 \\
1.4 \%\end{array}$ \\
\hline
\end{tabular}

Tabla 3 Percepción de los estudiantes para el uso de la aplicación de Realidad Aumentada.

Fuente: Elaboración propia, 2020

\section{Agradecimientos}

El presente estudio fue financiado con recursos propios de la Universidad Tecnológica de la Selva, a través de la Convocatoria Fomento a la Formación de Recursos Humanos de Alta Calidad y Desarrollo de Proyectos de Investigación. Por lo anterior, se extiende un agradecimiento a la misma por el apoyo brindado.

Por otro lado, se hace un agradecimiento especial a la Delegación Regional de la Secretaría de Educación Pública, quiénes proporcionaron información esencial para el proyecto, así como, a los Directores y Docentes de las 3 escuelas, por las facilidades proporcionadas para efectuar esta investigación.

Se hace un agradecimiento particular a la Lic. Guiomar Cameras Martínez, por las revisiones y correcciones realizadas a la traducción del resumen del presente artículo. 


\section{Conclusiones}

Los resultados obtenidos en el estudio demuestran que utilizar una herramienta como la realidad aumentada en el proceso de enseñanza y aprendizaje, los estudiantes obtienen mejores resultados en su rendimiento académico, comparado con los estudiantes que aprenden de manera tradicional. Otros estudios, también han demostrado que el rendimiento de los estudiantes mejora cuando en el proceso de enseñanza se utiliza una tecnología como ésta (López y Gutiérrez, 2018; Parroquín et al., 2013; Bursali y Yilmaz, 2019).

La percepción de los estudiantes que interactuaron con la aplicación de realidad aumentada, demuestra que el $73.6 \%$ le gustó muchísimo usar esta tecnología en clases para aprender, el $15.2 \%$ le gustó mucho, el $2.4 \%$ se mostró indiferente, $7.1 \%$ le gustó poco y el $1.7 \%$ no le gustó utilizarla.

Por lo anterior, se concluye que los docentes de educación básica (primaria y secundaria) deben hacer esfuerzos para incluir la tecnología en el quehacer educativo, ya que diversos estudios han demostrado que los estudiantes mejoran sus calificaciones al incluir una tecnología en el aula de clases, mejor aún, si se trata de una aplicación de realidad aumentada, ya que tiene la posibilidad de plantear escenarios que no pueden ser percibidos cotidianamente en el aula de clases, tales como, trabajar a fondo los diversos aparatos del cuerpo humano que las láminas o imágenes dentro de los libros de texto, no pueden especificar formas, tamaños o colores reales, por otro lado, trabajar el sistema solar, es muy difícil plantear este escenario en clases, mientras que una aplicación de realidad aumentada podría hacerlo.

A las autoridades educativas Estatales y Federales, se les exhorta a destinar recursos para la infraestructura tecnológica de manera equitativa a todas las regiones de los estados, con el fin de estar a la vanguardia internacional en el uso de las TIC en el aula de clases, asimismo, destinar recursos para la capacitación docente, para facilitar la implementación de las TIC en su labor docente, innovar el proceso de enseñanza y aprendizaje, y despertar el interés de los estudiantes en asistir a clases y utilizarlas en la construcción de su aprendizaje.

\section{Líneas abiertas de investigación}

La realización de este estudio deja abiertas algunas líneas de investigación para explorar, como la percepción de los docentes de educación básica para utilizar la realidad aumentada en el aula de clases, así como, el diseño de una secuencia didáctica para el uso de la realidad aumentada en la vida cotidiana del docente.

\section{Referencias}

Angarita, J. J. (2018). Apropiación de la realidad aumentada como apoyo a la enseñanza de las ciencias naturales en educación básica primaria. Boletín Redipe, 7(12), 144-157

Avendaño, V. C. (2015). Implementación y uso escolar de las tecnologías de la información y la comunicación en la Meseta Comitéca Tojolabal del estado de Chiapas. Primera Edición. México: División de Investigación y Posgrado, CRESUR.

Azuma, R. (1997). A Survey of Augmented Reality. In Presence: Teleoperators and Virtual Environments. 6, 4 August 1997, 355-385. Recuperado de https://www.cs.unc.edu/ azuma/ARpresence.pd $\mathrm{f}$

Bursali, H. y Yilmaz, R. M. (2019). Effect of augmented reality applications on secondary school students' reading comprehension and learning permanency, Comput. Human Behav. 95(2019), 126-135. https://doi.org/10.1016/j.chb.2019.01.035

Carrillo, J. L. y Cortés, J. A. (2016). Secuencias didácticas con realidad virtual: En el área de geometría en educación básica. F@ro: Revista Teórica del Departamento de Ciencias de la Comunicación, 1(23), 279-304

Domínguez, M. I. y Sandoval, M. (2017). ¿Lograrán las Tecnologías de la Información y Comunicación (TIC's) apoyar en la calidad educativa en las Instituciones de Educación Básica? En García, J. L. C., Barraza, I. y Jaik, A. (Edición 1, p. 52-53). México: Red Durango de Investigadores Educativos A. C. 
Gómez, I., Medel, R. y García, R. (2018). Realidad Aumentada como herramienta didáctica en geometría 3D. Latin-American Journal of Physics Education, 12(4), 4003-14003-8

Hamiyet, B. y Rabia, M. Y. (2019). Effect of Augmented Reality Applications on Secondary School Students' Reading Comprehension and Learning Permanency. Computers in Human Behavior, 15, 126-135. doi: 10.1016/j.chb.2019.01.035

Hernández, H. y Bautista, S. (2017). Las TIC en el sistema Educativo Mexicano. Revista Electrónica sobre Tecnología, Educación y Sociedad. 4(7). Recuperado de http://www.ctes.org.mx/index.php/ctes/article/v iew/656/742

Hernández Sampieri, R., Fernández Collado, C., y Baptista Lucio, M. (2014). Metodología de la investigación (Sexta edición). México: McGraw-Hill.

Jiménez, M. (2000) Competencia social: intervención preventiva en la escuela. Infancia y Sociedad. 24, 21-48.

Linares, G. (2014). Programa "Mi Compu.Mx": alfabetización digital para todos. Revista Iberoamericana de Producción Académica y Gestión Educativa. Publicación \#2. Recuperado de

https://www.pag.org.mx/index.php/PAG/article /viewFile/216/264

López, J. D., y Gutiérrez, D. (2018). Efecto del uso de la herramienta "realidad aumentada" en el rendimiento académico de estudiantes de Educación Básica. Revista Perspectivas, 3(1), 6-12. https://doi.org/10.22463/25909215.1464

Marín, V. (2018). La realidad aumentada al servicio de la Inclusión Educativa. Estudio de caso. Revista RETOS XXI, 2(1), 60-72. https://doi.org/10.33412/retoxxi.v2.1.2060

Marín, V. y Muñoz, V. P. (2018). Trabajar el cuerpo humano con realidad aumentada en educación infantil. Revista Tecnología, Ciencia y Educación, (9), 148-158
Merino, C., Pino, S., Meyer, E., Garrido, J. M. y Gallardo, F. (2015). Realidad aumentada para el diseño de secuencias de enseñanzaaprendizaje en química. Educación Química, 26(2), 94-99. https://doi.org/10.1016/j.eq.2015.04.004

Mirete, A. B. (2010). FORMACIÓN DOCENTE EN TICS. ¿ESTÁN LOS DOCENTES PREPARADOS PARA LA (R)EVOLUCIÓN TIC?. International Journal of Developmental and Educational Psychology, 4(1),35-44

Morales, M., Benítez, C., Silva, D., Altamirano, M. y Mendoza, H. M. (2015). Aplicación móvil para el aprendizaje del inglés utilizando realidad aumentada. Revista Iberoamericana de Producción Académica y Gestión Educativa, Recuperado de https://www.pag.org.mx/index.php/PAG/article /view/513

Ontoria, A. (2004). Aprendizaje centrado en el alumno (ACA) nueva mentalidad docente en la convergencia europea. ED.UCO: revista de investigación educativa, 1, 38-61

Osorio, M. C. (2012). Principales factores que determinan el bajo rendimiento en la escuela primaria (Tesis de Licenciatura). Universidad Pedagógica Nacional, Campeche, México.

Parroquín, P., Ramírez, J., González, V., y Mendoza, A. (2013). Aplicación de realidad aumentada en la enseñanza de la física. Cultura Científica y Tecnológica, 10(51), 182-192

Pérez, L. A., Flores, C., Tovar, S., Sánchez M. A., Ayala, I. J. y Sagaz, M. A. (2016). Técnicas aplicadas de realidad virtual y realidad aumentada para el fomento de la biodiversidad de áreas naturales protegidas de Querétaro. La Mecatrónica en México, 5(1), 10 - 15

Ruíz, C. J. (2013). Instrumentos y Técnicas de Investigación Educativa. Un Enfoque Cuantitativo y Cualitativo para la Recolección y Análisis de Datos. Tercera Edición. Editorial DANAGA Training and Consulting. Houston, Texas, USA.

Ruiz, D. (2011). Realidad Aumentada, Educación y Museos. REVISTA ICONO 14. 9(2), 212-226. 
Sáez, J. M., Cózar, R. y Domínguez, M. C. (2018). Realidad aumentada en Educación Primaria: comprensión de elementos artísticos y aplicación didáctica en ciencias sociales. Digital Education Review, 34, 59-75.

Secretaría General de Gobierno (2019). Plan de Desarrollo Estatal 2019-2024. Disponible en https://congresochiapas.gob.mx/legislaturalxvii/ files/Plan\%20Estatal\%20de\%20Desarrollo\%20 Chiapas\%202019-2024_opt.pdf

Van Krevelen, D., y Poelman, R. (2010). A survey of augmented reality technologies, applications and limitations. International Journal of Virtual Reality, 9(2), 1 - 20.

Vásquez, E. (s.f.). Las TIC en la educación pública de México, un esfuerzo disparejo. Recuperado de https://u-gob.com/las-tic-en-laeducacion-publica-de-mexico-un-esfuerzodisparejo/

Zarate, M. R., Mendoza, C. F., Aguilar, H. y Padilla, J. M. (2013). Marcadores para la Realidad Aumentada para fines educativos. ReCIBE. Revista electrónica de Computación, Informática, Biomédica y Electrónica, 2(3). Disponible en: https://www.redalyc.org/articulo.oa?id=5122/5 12251564004

Zorro, D. P., Torres, D. A. y Rivera, C. E. (2019). Realidad Aumentada como herramienta para la educación Socioambiental de estudiantes de 6to grado. (Tesis de Especialidad). Universitaria Agustiniana, Facultad de Humanidades, Ciencias Sociales y Educación. Bogotá, D.C. 